\title{
Transposons, Tandem Repeats, and the Silencing of Imprinted Genes
}

\author{
R. Martienssen, ${ }^{*}$ Z. Lippman, ${ }^{*}$ B. May, M. Ronemus, and M. Vaughn \\ Cold Spring Harbor Laboratory and "Watson School of Biological Sciences, Cold Spring Harbor, New York 11724
}

Most eukaryotic cells cleave double-stranded RNA (dsRNA) into small 21-24-bp RNA fragments via the RNAse III helicase Dicer (Hannon 2002). These small interfering RNA (siRNA) are unwound and loaded onto the PAZ domain of Argonaute proteins, where they guide the RNAseH-related PIWI domain to cleave single-stranded RNA complementary to the small RNA (Song et al. 2003, 2004; Liu et al. 2004). Cleavage sites can initiate secondstrand RNA synthesis via RNA-dependent RNA polymerase (RdRP) (Makeyev and Bamford 2002). Further processing of this dsRNA by Dicer leads to further (secondary) siRNA, which can reinitiate RdRP synthesis and thus amplify the cycle. RdRP can also incorporate siRNA directly into dsRNA by primer extension (Makeyev and Bamford 2002).

In the fission yeast Saccharomyces pombe, centromeric repeats are transcribed into dsRNA, which is subject to cleavage into siRNA and resynthesis, by Dicer, Argonaute, and RdRP, respectively (Volpe et al. 2002). However, while one strand of the outer repeats is continually transcribed, the other strand is transcriptionally silenced, as are reporter genes integrated within the repeats. This transcriptional silencing depends on the HP-1 homolog Swi6, which is recruited to the repeats by histone H3 lysine-9 dimethylation ( $\mathrm{H} 3 \mathrm{mK} 9)$ via a chromodomain (Bannister et al. 2001). Histone modification of the re-

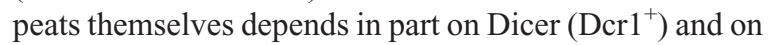
$\operatorname{RdRP}\left(\operatorname{Rdp}^{+}\right)$, and to a lesser extent on Argonaute $\left(\mathrm{Agol}^{+}\right)$, while histone modification of the reporter genes depends strongly on all three genes (Volpe et al. 2002). The accumulation of siRNA also depends on the histone lysine 9 methyltransferase $\mathrm{Clr}^{+}{ }^{+}$(Schramke and Allshire 2003), indicating a "reverse pathway" from histone modification to RNAi. Agol ${ }^{+}$is found in the RITS (RNA-induced initiation of transcriptional gene silencing) complex, along with $\mathrm{Chp1}^{+}+$(a chromodomain protein) and $\mathrm{Tas}^{+}$, as well as siRNA from heterochromatic repeats (Verdel et al. 2004). This complex was initially proposed to mediate initiation of transcriptional silencing via RNA interference (RNAi) (hence its name) but recent evidence suggests it may have a prominent role in spreading of silencing to reporter genes integrated nearby (Sigova et al. 2004), consistent with the substantial loss of $\mathrm{H} 3 \mathrm{mK} 9$ from the reporter genes (Volpe et al. 2002).

Argonaute is conserved in the higher plant, Arabidopsis, but the other members of the RITS complex, Chp $1^{+}$ and $\mathrm{Tas}^{+}$, have no close homologs in plants or generally in eukaryotes, respectively. siRNA, on the other hand, has been found corresponding to Arabidopsis centromeric repeats and transposons (Llave et al. 2002; Xie et al. 2004), both of which are also associated with histone H3 lysine 9 dimethylation (Gendrel et al. 2002; Johnson et al. 2002; Soppe et al. 2002; Lippman et al. 2004; Tariq and Paszkowski 2004). Unlike in yeast, cytosines are methylated in Arabidopsis transposons, both in CG and CXG di- and trinucleotides, as well as other noncanonical contexts. This methylation may mask some of the effects of RNAi on transcriptional silencing. Further, Dicer, Argonaute, and RdRP are encoded by multiple Arabidopsis genes, as are SET (Suppressor of variegation, Enhancer of Zeste, Trithorax) domain proteins (Lippman and Martienssen 2004). Even so, histone and DNA methylation are lost from a handful of transposons in agol and ago4, in which transposon siRNA is reduced, and in $d c l 3$ and $r d r 2$, in which siRNA is absent (Lippman et al. 2003; Zilberman et al. 2003; Xie et al. 2004).

Unlike in fission yeast, Arabidopsis siRNA are maintained in the absence of histone H3 lysine 9 dimethylation mediated by the methyltransferase KYP/SUVH4 (Lippman et al. 2003), reflecting either redundancy with other methyltransferases or the absence of RITS. However, some siRNA are lost in met 1 , which encodes a homolog of the mammalian CG methyltransferase dnmt1, and in $d d m 1$, which encodes a homolog of the mammalian SWI/SNF ATPase Lsh1 (Jeddeloh et al. 1999), both of which also lose histone H3mK9 (Gendrel et al. 2002; Johnson et al. 2002; Soppe et al. 2002; Lippman et al. 2003; Tariq et al. 2003). These results are consistent with the presence of a complex containing MET1, DDM1, and siRNA (Lippman et al. 2003). Some siRNA are lost in the dnmt3 double mutants drm 1 drm2, indicating that multiple pathways may include transposon siRNA (Lippman and Martienssen 2004; Matzke et al. 2004).

Here we review the role of siRNA and heterochromatin in transcriptional silencing of transposons and nearby genes in Arabidopsis. Transposons containing tandem repeats have more prevalent siRNA than other transposons and may contribute to silencing that depends on such repeats. The imprinted genes $M E D E A$ and $F W A$ are flanked by transposons and tandem repeats, and corresponding siRNA may be involved in silencing. A similar role for tandem repeats in mammalian cells could contribute to imprinting as well as silencing mediated by triplet repeats. 


\section{SILENCING OF ROBERTSON'S MUTATOR TRANSPOSONS IN ARABIDOPSIS}

Most transposable elements (TEs) in Arabidopsis remain silent and methylated in the RNA interference mutants $d c l 3, r d r 2$, agol, and ago4, as well as in the histone H3 lysine-9 methyltransferase mutant $k y p$, indicating that silencing can be maintained in the absence of RNAi (Lippman and Martienssen 2004; Xie et al. 2004). An exception is AtSN1, a SINE element that has three- to eightfold elevated expression levels and loses $\sim 50 \%$ of associated histone $\mathrm{H} 3$ methylated on lysine- 9 in mutants in RNAi (Xie et al. 2004). However, most TEs do not exhibit even these modest changes. The Robertson's Mutator $(M u)$ elements are found in Arabidopsis as they are in maize, and full-length elements, capable of autonomous transposition, have been found at low copy numbers in many different plant genomes. An Arabidopsis $M u$, AtMu1, is located on chromosome 4L, just distal to the pericentromeric heterochromatin, where it is associated with both histone $\mathrm{H} 3 \mathrm{~K} 9$ methylation and histone $\mathrm{H} 3 \mathrm{~K} 4$ methylation (Fig. 1). H3K9 methylation is lost in met1 and $d d m 1$ mutants, while $\mathrm{H} 3 \mathrm{~K} 4$ methylation is enriched. These histone modifications remain unchanged in the chromodomain methyltransferase mutant $\mathrm{cmt3}$, which impacts CXG and non-CG methylation (Fig. 1) (Lippman et al. 2003). DNA methylation at AtMul was measured using semiquantitative polymerase chain reaction (PCR) following digestion with the methylation-dependent restriction enzyme mcrBC, and was reduced in each of the mutants (Fig. 1B).

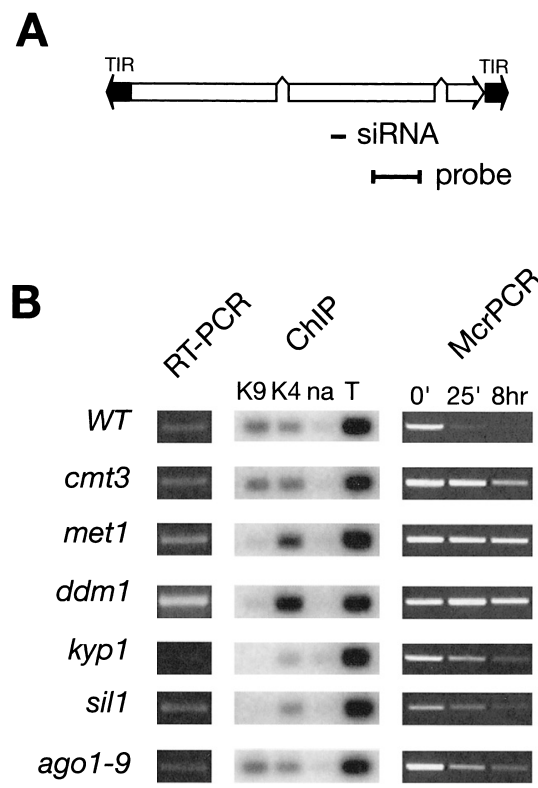

AtMul germinal transposition frequencies were elevated more than 20 -fold in $d d m 1$, and transposed elements were found all over the genome (Singer et al. 2001). In contrast, transposition frequencies were elevated only two- to threefold in metl (data not shown) despite loss of histone H3 lysine 9 methylation and CG DNA methylation, and a substantial increase in transposase gene expression (Fig. 1B). In cmt3 met1 double mutants, however, transposition could be readily detected on Southern blots (Fig. 1C). Transposition of the CACTA elements $C A C 1$ and $C A C 2$ is similarly regulated (Kato et al. 2003). One explanation is that met 1 and cmt3 act in distinct pathways to regulate transposons, perhaps reflecting distinct methylation-sensitive DNA-binding sites in AtMul for transposase on the one hand and the transcriptional machinery on the other. In this way MET1 might regulate silencing, while CMT3 regulates transposition. Transcripts are only slightly elevated in $\mathrm{cmt} 3$ relative to met1, supporting this idea (Lippman et al. 2003).

Levels of AtMul RNA are also slightly elevated in agol, which encodes one of ten argonaute homologs in Arabidopsis and is likely to bind siRNA (Song et al. 2003). We therefore examined levels of siRNA corresponding to AtMul in wild type (WT) as well as in mutant strains defective in RNAi, histone, and DNA modification (Lippman et al. 2003). We found low but detectable levels of siRNA in WT Ler strains, as well as in ago1, the histone deacetylase sill, cmt3, and kyp (Lippman et al. 2003), but they failed to accumulate in the dicer mutant $d c l 3$, as well as in the RdRP mutant $r d r 2$ (Fig. 1D). These mutants fail to accumulate siRNA from most

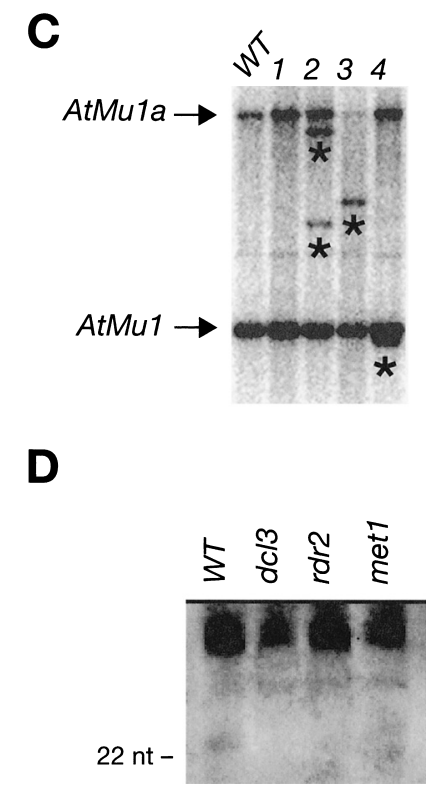

Figure 1. Silencing and transposition of Mu transposon AtMu1. (A) The Arabidopsis AtMul element on chromosome 4 has long terminal inverted repeats (TIRs), but cloned small interfering RNA (siRNA) corresponds to the transposase gene itself. Adjacent probes (shown) were used to detect siRNA on northern blots. (B) AtMul transcripts (RT-PCR [reverse transcriptase polymerase chain reaction]), associated histone modifications (ChIP[chromatin immunoprecipitation]), and DNA methylation (McrPCR) were detected by PCR amplification using primers from the region shown in $A$. A time course of predigestion with mcrBC is shown. $(C)$ Transposed AtMul elements could be detected only at low frequency in wild type (WT) $(1 \%)$, met1 $(6 \%)$, and cmt $3(2 \%)$, but in high frequency $(25 \%)$ in cmt 3 met 1 double mutants (lanes $1-4$ ). Loss of a band (lane 3 ) most likely represents excision. (D) siRNA from AtMul could be detected with the probe shown in $A$ in WT, but not in $d c l 3, r d r 2$, or met 1 mutant plants. 
if not all other transposons, indicating they are specific for 24-nt heterochromatic siRNA (Xie et al. 2004). Interestingly, AtMul siRNA also failed to accumulate in met 1 and $d d m 1$ both in Columbia (Fig. 1) and in the Landsberg background (Lippman et al. 2003). Many, though not all heterochromatic siRNA, are lost in met1 (Lippman et al. 2004). One possibility is that RNAi of these sequences depends on heterochromatic modification (Lippman and Martienssen 2004). Alternatively, MET1 might form a complex with some siRNA to guide it to specific sequences (Lippman et al. 2003).

No changes in histone modification were detectable in agol using probes from the terminal inverted repeat (TIR) (Lippman et al. 2003). However, DNA methylation was detectably reduced in these same repeats (Fig. 1) indicating a weak role for RNAi in their modification (Lippman et al. 2003). Comparable levels of demethylation (loss of one methylated cytosine out of three tested) has been reported for AtMul TIR in ago4 (Zilberman et al. 2003), raising the possibility that AGO1 and AGO4 might have partially overlapping functions.

Two closely related Mutator elements, AtMula and AtMulb, are found on chromosomes 5L and 1L, respectively, in the Columbia background (Singer et al. 2001). Both elements are heavily methylated and do not trans- pose. In Landsberg erecta, AtMula has transposed to the short arm of chromosome 1 and is transcribed but transposes at a very low frequency (data not shown).

\section{TANDEM REPEATS}

In addition to transposons, heterochromatic tandem repeats in Arabidopsis are found in pericentromeric domains, nucleolar organizers, and in the interstitial knobs on chromosomes 4 and 5 (Arabidopsis Genome Initiative 2000; CSHL/WUGSC/PEB Arabidopsis Sequencing Consortium 2000; Tabata et al. 2000). The interstitial knob repeats on chromosomes 4 and 5 are derived from CACTA and Mutator class transposons, respectively, indicating that the arrangement of the repeats, in addition to their sequence, gives rise to heterochromatic modification.

We examined the correlation between tandem repeats, transposons, and small RNAs in the Arabidopsis genome (Fig. 2a). Tandem repeats were discovered using "Tandem Repeats Finder" (Benson 1999) and transposon-like repeats were annotated using CENSOR/Repbase (Jurka et al. 1996). Sequence homologies to siRNA were determined by BLASTN analysis of Arabidopsis Small RNA Project database sequences cloned from WT plants (Xie et al. 2004). We excluded microRNAs, which are derived

a)

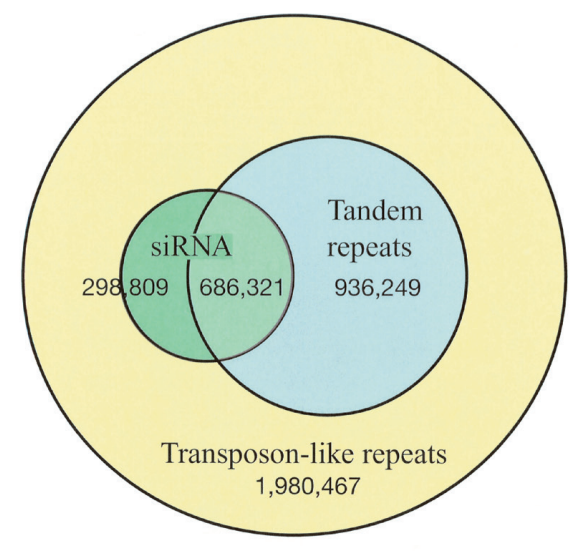

b)

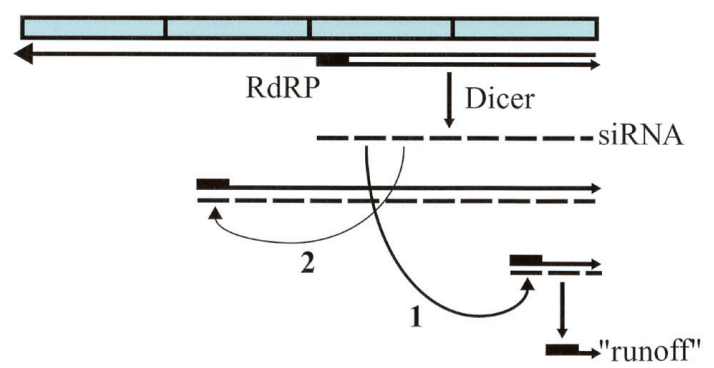

Figure 2. Tandem repeats and small interfering RNA (siRNA). (a) Tandem repeats significantly influence the abundance of siRNA in Arabidopsis. Approximately one third of transposon-like repeat sequences contained tandem repeats, and one third had siRNA homology by BLASTN. Of the sequences matching siRNA, two thirds were found in conjunction with tandem repeats, suggesting that the presence of tandem repeats within a given transposon enhances the generation of small RNAs. $(b)$ Long tandem repeats could maintain populations of small RNA if they are transcribed from one strand. This is because siRNA can initiate multiple rounds of second-strand synthesis both upstream and downstream of their origin in the sequence (arrow 2). Short tandem arrays also generate siRNA, but these lead to longer "runoff" products (arrow 1) when they initiate second-strand synthesis downstream that are too short for Dicer digestion (Martienssen 2003). Initiation of second-strand synthesis can occur either by primer extension or by cleavage by Argonaute, followed by initiation at the cleavage site. Both depend on siRNA. 
from hairpin precursor genes (Hannon 2002). Each transposon-like repeat in a $42-\mathrm{Mb}$ sample of euchromatic sequence was assayed by bioinformatic means for presence of siRNA homology and correspondence to annotated tandem repeats. Results were tabulated and expressed as total base pair lengths per logical category. Transposon-like repeats accounted for $2.92 \mathrm{Mb}$ of the sampled sequence $(7 \%)$. Of this transposon-like sequence, almost a third $(0.94 \mathrm{Mb})$ included tandem repeats. Randomly sequenced siRNA corresponded to $73 \%$ of transposon sequences containing tandem repeats, but only $15 \%$ of transposon sequences lacking them. This strongly implicates tandem repeats in influencing the abundance of siRNA.

When repeats are arranged in tandem, secondary siRNA generated by Dicer can match repeats upstream as well as downstream of the primary siRNA used to initiate second-strand synthesis by RdRP (Fig. 2b). In this way, iterative rounds of siRNA-dependent RdRP synthesis can be maintained along with the population of siRNA (Martienssen 2003). Dispersed repeats that match an siRNA will not have this property. Instead, with each round of RdRP and Dicer activity, secondary siRNA, derived from dsRNA, will always lie downstream of the primary siRNA in each round of second-strand synthesis. The RdRP-generated transcripts will become shorter and shorter. Finally, RdRP will generate products that are no longer processed by Dicer, and these will accumulate instead of the siRNA (Martienssen 2003). The ability of a tandemly repeated array to reiterate second-strand synthesis in this way is expected to depend on the number of repeats, so that longer arrays will be more stably silenced than shorter ones (Fig. 2B).

\section{SILENCING IMPRINTED GENES BY TRANSPOSONS AND TANDEM REPEATS}

Chromosomal imprints are imposed early in development, but inherited through multiple mitotic divisions long after the imprinting signal has gone (Martienssen 1998a; Alleman and Doctor 2000). When imprinting occurs in one germ line but not the other, monoallelic gene expression results following fertilization. Imprinting occurs in seed plants and placental mammals and is thought to mediate conflicting parental interests in viviparous embryonic growth, as well as proliferation of extraembryonic tissues - namely, the placenta and the endosperm (Alleman and Doctor 2000; Sleutels and Barlow 2002). Chromosomal imprinting involves epigenetic modifications, such as histone $\mathrm{H} 3$ lysine- 9 methylation, as well as DNA methylation in plants and mammals (Adams et al. 2000; Sleutels and Barlow 2002). However, imprinting was first discovered in insects (Mohan et al. 2002), and related phenomena occur in Drosophila (Joanis and Lloyd 2002), which do not bear live offspring in this way, implicating a more ancient function.

In plants, imprinting is mostly, or entirely, limited to the endosperm, which is a terminally differentiated tissue, and so (unlike in animals) imprinting does not need to be reset during meiosis (Alleman and Doctor 2000). One example is the $F W A$ gene on chromosome 4. This gene encodes a homeodomain protein that is mono-allelically expressed in the endosperm from the maternal chromosome (Kinoshita et al. 2004). In the body of the plant, $F W A$ is silenced by MET1 and DDM1, and silencing is associated with both DNA methylation and histone H3 lysine 9 methylation (Soppe et al. 2000; Johnson et al. 2002; Lippman et al. 2004). In met1 mutants, ectopic expression of $F W A$ delays flowering, and late flowering epialleles of $F W A$ are subsequently inherited independently of met1 (Soppe et al. 2000).

Reannotation of the $F W A$ tandem repeats has revealed that they are part of a short interspersed nucleotide element (SINE) and were likely generated by reiterated integration of the transposon (Lippman et al. 2004). The SINE provides the $F W A$ promoter and is therefore responsible for silencing. siRNA corresponding to the SINE could be detected in WT and $d d m 1$, but not in met1. This could account for the high frequency of heritable epialleles of $F W A$ that arise in met1, if silencing cannot be reestablished in the absence of siRNA. Consistent with these results, transgenic $F W A$ is rapidly silenced in WT plants, but not in the RNAi mutants $d c l 3, r d r 2$, or ago4, resulting in late flowering phenotypes (Chan et al. 2004; Xie et al.. 2004). Presumably, these mutants have lost the siRNA.

The MEDEA locus on chromosome 1 is also imprinted in the endosperm, such that expression is limited to the central cell in the maternal gametophyte (before fertilization) and to the maternal allele in the endosperm in the first few days after fertilization (Grossniklaus et al. 1998; Luo et al. 2000). mea gametophytes give rise to aborted embryos if fertilized (Grossniklaus et al. 1998) and to proliferation of the endosperm if left unfertilized (Luo et al. 2000). DNA methylation influences imprinting in both plants and animals (Adams et al. 2000), but in plants the influence seems to be indirect (Vielle-Calzada et al. 1999; Luo et al. 2000). The DNA glycosylase DEMETER is required to activate maternal transcription of $M E A$ in WT, but not in met1, suggesting that DNA methylation is responsible for silencing (Choi et al. 2002; Xiao et al. 2003). MET1-dependent DNA methylation has been detected in two or three regions upstream of $M E A$, depending on genetic background, as well as in the $3^{\prime}$ region (Xiao et al. 2003; Zilberman et al. 2003). A 4.5-kb-upstream sequence is sufficient to restrict reporter gene expression to the maternal allele (Xiao et al. 2003).

Reannotation of the MEA locus reveals that the $5^{\prime}$ and 3 ' methylated regions correspond to two clusters of unrelated tandem repeats, $\sim 4 \mathrm{~kb}$ upstream and $3 \mathrm{~kb}$ downstream, respectively (Fig. 3). The upstream repeat (330 bp) is part of a 554-bp helitron transposon (ATREP2) while the downstream repeat $(1 \mathrm{~kb})$ is of unknown origin. siRNA corresponding to both repeats was detected in WT plants and was greatly reduced in met 1 and $d d m 1$ (Fig. 4). Interestingly, probes from the upstream repeat detected both 24-nucleotide siRNA and longer small RNA $>40$ nucleotides. These longer RNA did not change in met 1 , but were absent in $d d m 1$. Longer RNA may correspond to the terminal "runoff" products of second-strand synthesis by RdRP, which are predicted to accumulate in short tandem arrays (Fig. 2). This is because extension by RdRP near 

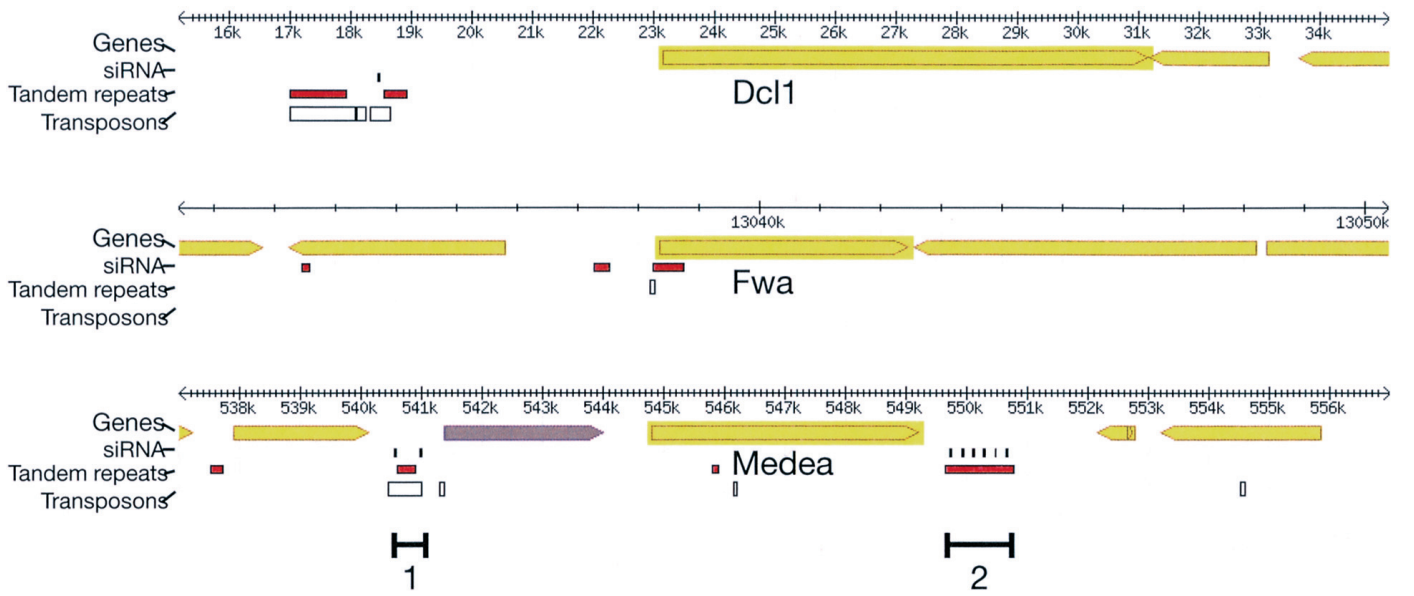

Figure 3. Tandem repeats and transposons surrounding imprinted loci in Arabidopsis. The genomic context for SIN1/DCL1 and for the imprinted genes, $F W A$ and $M E D E A$, are shown along with flanking repeats, transposons, and cloned siRNA. Probes for small RNA blots shown in Fig. 4 are indicated for $M E D E A$. Tandem repeats are found in each of the upstream regions required for imprinted expression and are generated by transposable elements.

the edges of the array will result in dsRNA products that are too short to be processed further by DICER (Martienssen 2003). In long tandem arrays, such as the one downstream of $M E A$, small RNA from central repeats are continually regenerated following extension (Fig. 2), so that longer products accumulate to a lesser degree (Fig. 4). This downstream repeat (also known as MEA-ISR) is heavily methylated, and non-CG methylation depends on $A G O 4$, although this is not the case at $F W A$ (Zilberman et al. 2003).

\section{SILENCING MECHANISMS}

Silencing of transposons and transgenes in Arabidopsis depends on at least three hierarchical mechanisms, one involving MET1, DDM1, and the histone deacetylase SIL1, another involving histone methylation and the chromodomain methyltransferase CMT3, and a third that utilizes the dnmt3 homologs DRM1 and DRM2 (Cao et al. 2003; Lippman et al. 2003; Matzke et al. 2004). RNAi has been implicated in all three mechanisms (Lippman and Martienssen 2004) and each complex has partially overlapping roles. For example, the chromomethylase CMT3 plays a role in transcriptional silencing via the histone modification pathway, consistent with recruitment to methylated histones via the chromodomain (Bartee et al. 2001; Jackson et al. 2002; Johnson et al. 2002). However, CMT3 also has a role in transposition of AtMul and CACTA-class DNA transposons (Kato et al. 2003). In contrast, MET1 alone has a major role in transcriptional silencing, without effecting transposition, while DDM1 affects both (Miura et al. 2001; Singer et al. 2001).

AtMul siRNA, whether detected by northern analysis or as cloned fragments, is derived from the transposase gene (Fig. 1). In this respect it differs from transposon $T c 1$ siRNA in Caenorhabditis elegans, which corresponds to

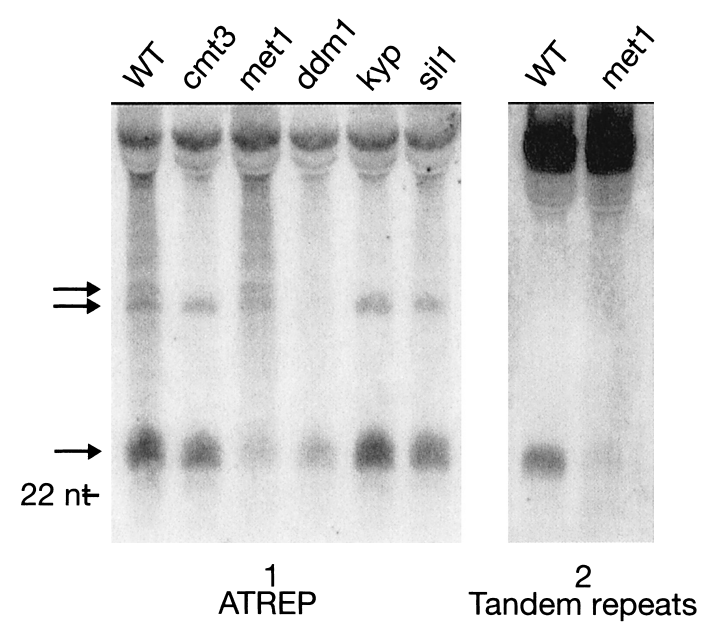

Figure 4. Small RNA corresponding to tandem repeats at MEDEA. siRNA corresponding to the upstream ATREP helitron transposon (1) and downstream MEA-ISR (2) repeats can be detected in WT, in the histone K9 methyltransferase mutant kyp, in the histone deacetylase mutant sill, and in the chromomethylase mutant cmt3. They are substantially reduced in met 1 and $d d m 1$. Longer short RNA (arrows) is found only in the upstream repeats and depends on DDM1. 
long TIRs. It has been proposed that $T c 1$ siRNA is derived from dsRNA by read-through transcription starting outside the element and foldback of the resulting transcript (Sijen and Plasterk 2003). Another origin might be found in extrachromosomal circles, which are found in both maize and C. elegans (Ruan and Emmons 1984; Sundaresan and Freeling 1987). Transcription of these circles would generate hairpin RNA from the TIR, which could act as a potential source of siRNA. Retrotransposons generate siRNA corresponding to the LTR repeats (Lippman et al. 2003). This is important, because transposon promoters are encoded within these repeats and can therefore be subject to transcriptional silencing when targeted by siRNA. Silencing targeted to the coding region of AtMul, by contrast, would have to spread to the promoter to fully silence transcription, accounting perhaps for the low mRNA levels observed in WT plants (Fig. 1B). Robertson's Mutator transposons in maize also correspond to siRNA, although its role in silencing is unclear (Rudenko et al. 2003).

In addition to providing promoters for themselves, transposons provide promoters for neighboring genes. For example, SINEs provide promoters and exons for neighboring genes in mammalian genomes (Allen et al. 2004) as well as in Arabidopsis (Lippman et al. 2004). Both DNA-class and retrotransposable elements provide promoters for neighboring genes in animals, plants, yeast, and filamentous fungi, as well as in prokaryotes (Martienssen 1998b; Martienssen and Colot 2001). This may be a fundamental property of transposons, in accordance with their role as controlling elements first envisioned by McClintock (1957). Transposon promoters are regulated by transposon proteins, including transposase itself. In Arabidopsis, the regulatory proteins FAR1 and FHY3 each closely resemble the maize Mutator transposase Jittery and presumably regulate genes via fragments of defective elements integrated in their promoters (Hudson et al. 2003). Unlike other Mutator elements, this family of related genes is largely unmethylated (R.A. Martienssen et al., unpubl.).

Tandem repeats are a common constituent of most eukaryotic genomes. In the human genome, they are highly polymorphic and are often generated by insertion of SINE elements, much like the repeats at FWA (Bois et al. 1998). In general, such polymorphisms are neutral, but occasionally short tandem repeats arise in genes. At FMR1, a gene responsible for Fragile $\mathrm{X}$ syndrome, expansion of a triplet CGG tandem repeat from 20 to 60 or more copies in the untranslated leader leads to DNA and histone H3 lysine-9 methylation, gene silencing, and sexlinked disease (Jin and Warren 2000; Coffee et al. 2002). It is possible that silencing in this case results from the mechanism described here, given that siRNA induces transcriptional silencing in humans as well as plants (Morris et al. 2004). FMR repeats are transcribed, and siRNA could be maintained if sufficient copies of the repeat were present to permit reiterative rounds of secondstrand synthesis and degradation. Sixty copies of a triplet repeat would correspond to seven sequential 24-nucleotide repeats in three different registers, providing a rich source of siRNA. Conversely, loss of tandem repeats would be expected to result in loss of gene silencing, ectopic expression, and autosomal dominant disease.

Tandem repeat and transposon-mediated silencing are attractive mechanisms for imprinting. This is because transcription of the repeats in the germ line would establish silencing in the next generation, which could then be maintained in cis by epigenetic modifications. Expression of tandem repeats in the female germ line would result in silencing of the maternal allele, while expression in the male germ line would result in paternal chromosome silencing. Tandem repeats have been found associated with many imprinting control regions in the mouse, although only some of them have been implicated in silencing (Lewis et al. 2004). They have also been found associated with imprinted and paramutable genes in maize (Alleman and Doctor 2000; Stam et al. 2002). In the case of $F W A$, transposon-derived tandem repeats regulate silencing and correspond to siRNA (Lippman et al. 2004). RNAi is required for establishment of silencing when mutant plants are transformed, but it is not required to maintain silencing, as $F W A$ remains silent in $d c l 3$ and $r d r 2$. This is consistent with a role for RNAi in establishing imprints in the germ line (Chan et al. 2004). Alternatively, RNAi could contribute to silencing throughout the plant and might be countered in the endosperm only by other mechanisms (Xiao et al. 2003).

In addition to 24-nucleotide siRNA, longer 40-80nucleotide products accumulate from the $M E A$ upstream tandem repeats ( 2.5 copies), and these depend on DDM1 but not MET1 (Fig. 4). Longer products are predicted to accumulate from shorter tandem repeat arrays as "runoff" products of second-strand synthesis (Fig. 2). It is therefore tempting to speculate that DDM1 may play a role in second-strand RNA synthesis and only indirectly in the synthesis of siRNA. As well as having chromatin remodeling activity (Brzeski and Jerzmanowski 2003), DDM1 is a DEAD box helicase (Jeddeloh et al. 1999) and may have a role in RNA metabolism.

In Arabidopsis, DNA methylation has a major role in silencing of the imprinted genes $M E D E A$ and $F W A$. The DNA glycosylase DEMETER acts in opposition to MET1 to activate $M E A$ and $F W A$ in the central cells of the female gametophyte, which go on to form the extraembryonic endosperm (Xiao et al. 2003; Kinoshita et al. 2004). Activation is inherited for a few cell divisions following fertilization, so that the maternal allele is expressed in the early endosperm. Maternal mea seed abortion cannot be rescued when met 1 is transmitted through the pollen, consistent with a role for MEA and MET1 in pre- but not postfertilization (Xiao et al. 2003). DDM1 does not effect imprinting directly (Vielle-Calzada et al. 1999; Xiao et al. 2003) and may not be functional in the gametophyte, unlike MET1 (Vongs et al. 1993; Saze et al. 2003).

In plants, DNA methylation is targeted to transposable elements (Rabinowicz et al. 2003), which implicates transposons in imprinting, as well as other silencing mechanisms (Martienssen 1998a,b). Imprinted genes were first identified in maize, and at least some imprinted alleles have transposon sequences within their promoters (Alleman and Doctor 2000). As well as FWA and $M E A$, 
methylated repeats are found upstream of a third gene, SHORT INTEGUMENTS1 (SIN1) which has a tandem repeat $3.5 \mathrm{~kb}$ upstream (Fig. 3). This upstream region directs maternal monoallelic reporter gene expression in transgenic plants (Golden et al. 2002). SIN1 encodes the dicer-like gene $D C L 1$. The methylated repeats upstream of $F W A, M E A$, and DCL1 are derived from a 422-bp SINE element, a 554-bp helitron, and a 1186-bp helitron, respectively, each of which corresponds to siRNA (Fig. 3). Loss of SINE and helitron siRNA in met1 implicates RNAi in silencing and indicates transposons may regulate imprinted genes (Lippman et al. 2004). Some retrotransposons in mammals are also imprinted (Peaston et al. 2004) and can influence gene expression depending on parent of origin (Morgan et al. 1999). The finding of noncoding RNA at differentially methylated regions of imprinted loci in the mouse suggests RNA-mediated mechanisms may play a role (Sleutels et al. 2002).

If siRNA mediates transposon silencing, why do most transposons remain silent in mutants in RNAi? In Arabidopsis, redundancy among RNAi genes may explain this, but retrotransposons also remain largely silent in fission yeast RNAi mutants (Hansen et al. 2004), even though some lose H3mK9 (Schramke and Allshire 2003). One explanation is that RNAi is required only for initiation of silencing and plays no further role once silencing is established. Although this is not the case at the centromere (Volpe et al. 2002), it could be the case at the mating-type locus (Hall et al. 2002). However, recent studies indicate this is not the entire explanation. First, maintenance of silencing at the mating-type locus does depend on RNAi after all (as it does at the centromere), but only in the absence of certain transcription factors (Jia et al. 2004). Second, the RITS complex remains bound to the mating type locus in $d c r 1^{-}$, but not in $c l r 4^{-}$(Sigova et al. 2004), indicating $\mathrm{H} 3 \mathrm{mK} 9$ is required for binding but siRNA is not. Instead of initiation, RITS might be involved in spreading of heterochromatic silencing, as loss of $\mathrm{H} 3 \mathrm{mK} 9$ from centromeric reporter genes is more severe in RITS mutants than loss from neighboring heterochromatic repeats (Volpe et al. 2002; Sigova et al. 2004).

Nonetheless, RNAi is required to initially silence $F W A$ transgenes in Arabidopsis following infection of the female gametophyte with Agrobacterium (see above), while it is not required to maintain silencing of the endogenous FWA gene (Chan et al. 2004). Similarly, epialleles of $F W A$ are inherited from met1, which loses siRNA, but not from $d d m 1$, which does not, implicating siRNA in transposon-mediated silencing in cis (Lippman et al. 2004). Further genetic analysis should clarify this issue in plants as well as in fission yeast.

\section{CONCLUSION}

Heterochromatin is composed primarily of transposons and related repeats. In fission yeast and plants, at least, these repetitive sequences correspond to siRNA, which helps to guide heterochromatic modifications to these regions of the chromosome. Tandem repeats can maintain large populations of siRNA and are frequently associated with epigenetic regulation, such as imprinting, paramutation, and centromere activity. Imprinted genes in Arabidopsis, such as FWA and MEDEA, are associated with tandem repeats, transposable elements, and siRNA, which are coregulated by the DNA methyltransferase MET1. Transposons and siRNA are thus implicated in imprinting and developmental control.

\section{ACKNOWLEDGMENTS}

We would like to thank Bob Fischer, Jim Carrington, Steve Jacobsen, and Greg Hannon for helpful discussions, as well as our colleagues Anne-Valerie Gendrel, Tom Volpe, and especially Vincent Colot for sharing their data and their advice. M.V. is a National Science Foundation bioinformatics postdoctoral fellow and Z.L. is an Arnold and Mabel Backman graduate fellow in the Watson School of Biological Sciences. M.R. has a postdoctoral fellowship from NIH. This work was supported by NSF grant DBI 000074, and NIH grant R01-067014 to R.M.

\section{REFERENCES}

Adams S., Vinkenoog R., Spielman M., Dickinson H.G., and Scott R.J. 2000. Parent-of-origin effects on seed development in Arabidopsis thaliana require DNA methylation. Development 127: 2493.

Alleman M. and Doctor J. 2000. Genomic imprinting in plants: Observations and evolutionary implications. Plant Mol. Biol. 43: 147.

Allen T.A., Von Kaenel S., Goodrich J.A., and Kugel J.F. 2004. The SINE-encoded mouse B2 RNA represses mRNA transcription in response to heat shock. Nat. Struct. Mol. Biol. 11: 816.

Arabidopsis Genome Initiative. 2000. Analysis of the genome sequence of the flowering plant Arabidopsis thaliana. Nature 408: 796.

Bannister A.J., Zegerman P., Partridge J.F., Miska E.A., Thomas J.O., Allshire R.C., and Kouzarides T. 2001. Selective recognition of methylated lysine 9 on histone H3 by the HP1 chromo domain. Nature 410: 120.

Bartee L., Malagnac F., and Bender J. 2001. Arabidopsis cmt3 chromomethylase mutations block non-CG methylation and silencing of an endogenous gene. Genes Dev. 15: 1753.

Benson G. 1999. Tandem repeats finder: A program to analyze DNA sequences. Nucleic Acids Res. 27: 573.

Bois P., Williamson J., Brown J., Dubrova Y.E., and Jeffreys A.J. 1998. A novel unstable mouse VNTR family expanded from SINE B1 elements. Genomics 49: 122.

Brzeski J. and Jerzmanowski A. 2003. Deficient in DNA methylation 1 (DDM1) defines a novel family of chromatin-remodeling factors. J. Biol. Chem. 278: 823.

Cao X., Aufsatz W., Zilberman D., Mette M.F., Huang M.S., Matzke M., and Jacobsen S.E. 2003. Role of the DRM and CMT3 methyltransferases in RNA-directed DNA methylation. Curr. Biol. 13: 2212.

Chan S.W., Zilberman D., Xie Z., Johansen L.K., Carrington J.C., and Jacobsen S.E. 2004. RNA silencing genes control de novo DNA methylation. Science 303: 1336.

Choi Y., Gehring M., Johnson L., Hannon M., Harada J.J., Goldberg R.B., Jacobsen S.E., and Fischer R.L. 2002. DEMETER, a DNA glycosylase domain protein, is required for endosperm gene imprinting and seed viability in Arabidopsis. Cell 110: 33.

Coffee B., Zhang F., Ceman S., Warren S.T., and Reines D. 2002. Histone modifications depict an aberrantly heterochromatinized FMR1 gene in fragile x syndrome. Am. J. Hum. Genet. 71: 923.

CSHL/WUGSC/PEB Arabidopsis Sequencing Consortium. 2000. The complete sequence of a heterochromatic island from a higher eukaryote. Cell 100: 377. 
Gendrel A.V., Lippman Z., Yordan C., Colot V., and Martienssen R.A. 2002. Dependence of heterochromatic histone $\mathrm{H} 3$ methylation patterns on the Arabidopsis gene DDM1. Science 297: 1871.

Golden T.A., Schauer S.E., Lang J.D., Pien S., Mushegian A.R., Grossniklaus U., Meinke D.W., and Ray A. 2002. SHORT INTEGUMENTS1/SUSPENSOR1/CARPEL FACTORY, a Dicer homolog, is a maternal effect gene required for embryo development in Arabidopsis. Plant Physiol. 130: 808.

Grossniklaus U., Vielle-Calzada J.P., Hoeppner M.A., and Gagliano W.B. 1998. Maternal control of embryogenesis by MEDEA, a polycomb group gene in Arabidopsis. Science 280: 446.

Hall I.M., Shankaranarayana G.D., Noma K., Ayoub N., Cohen A., and Grewal S.I. 2002. Establishment and maintenance of a heterochromatin domain. Science 297: 2232.

Hannon G.J. 2002. RNA interference. Nature 418: 244.

Hansen K.R., Burns G., Mata J., Volpe T.A., Martienssen R.A., Bahler J., and Thon G. 2005. Global effects on gene expression in fission yeast by silencing and RNA interference machineries. Mol. Cell. Biol. 25: 590.

Hudson M.E., Lisch D.R., and Quail P.H. 2003. The FHY3 and FAR1 genes encode transposase-related proteins involved in regulation of gene expression by the phytochrome A-signaling pathway. Plant J. 34: 453.

Jackson J.P., Lindroth A.M., Cao X., and Jacobsen S. 2002. Control of CpNpG DNA methylation by the KRYPTONITE histone H3 methyltransferase. Nature 416: 556.

Jeddeloh J.A., Stokes T.L., and Richards E.J. 1999. Maintenance of genomic methylation requires a SWI2/SNF2-like protein. Nat. Genet. 22: 94.

Jia S., Noma K., and Grewal S.I. 2004. RNAi-independent heterochromatin nucleation by the stress-activated ATF/CREB family proteins. Science 304: 1971.

Jin P. and Warren S.T. 2000. Understanding the molecular basis of fragile X syndrome. Hum. Mol. Genet. 9: 901.

Joanis V. and Lloyd V.K. 2002. Genomic imprinting in Drosophila is maintained by the products of Suppressor of variegation and trithorax group, but not Polycomb group, genes. Mol. Genet. Genomics 268: 103 .

Johnson L., Cao X., and Jacobsen S. 2002. Interplay between two epigenetic marks. DNA methylation and histone $\mathrm{H} 3 \mathrm{ly}$ sine 9 methylation. Curr. Biol. 12: 1360.

Jurka J., Klonowski P., Dagman V., and Pelton P. 1996. CENSOR: A program for identification and elimination of repetitive elements from DNA sequences. Comput. Chem. 20: 119.

Kato M., Miura A., Bender J., Jacobsen S.E., and Kakutani T. 2003. Role of CG and non-CG methylation in immobilization of transposons in Arabidopsis. Curr. Biol. 13: 421.

Kinoshita T., Miura A., Choi Y., Kinoshita Y., Cao X., Jacobsen S.E., Fischer R.L., and Kakutani T. 2004. One-way control of FWA imprinting in Arabidopsis endosperm by DNA methylation. Science 303: 521.

Lewis A., Mitsuya K., Constancia M., and Reik W. 2004. Tandem repeat hypothesis in imprinting: Deletion of a conserved direct repeat element upstream of H19 has no effect on imprinting in the Igf2-H19 region. Mol. Cell. Biol. 24: 5650.

Lippman Z. and Martienssen R. 2004. The role of RNA interference in heterochromatic silencing. Nature 431: 364.

Lippman Z., May B., Yordan C., Singer T., and Martienssen R. 2003. Distinct mechanisms determine transposon inheritance and methylation via small interfering RNA and histone modification. PLoS Biol. 1: E67.

Lippman Z.L., Gendrel A.V., Black M., Vaughn M.W., Dedhia D., McCombie W.R., Lavine K., Mittal V., May B., Kasschau K.D., Carrington J.C., Doerge R.W., Colot V., and Martienssen R.A. 2004. Role of transposable elements in heterochromatin and epigenetic control. Nature 430: 471.

Liu J., Carmell M.A., Rivas F.V., Marsden C.G., Thomson J.M., Song J.J., Hammond S.M., Joshua-Tor L., and Hannon G.J. 2004. Argonaute 2 is the catalytic engine of mammalian RNAi. Science 305: 1437.

Llave C., Kasschau K.D., Rector M.A., and Carrington J.C.
2002. Endogenous and silencing-associated small RNAs in plants. Plant Cell 14: 1605.

Luo M., Bilodeau P., Dennis E.S., Peacock W.J., and Chaudhury A. 2000. Expression and parent-of-origin effects for FIS2, MEA, and FIE in the endosperm and embryo of developing Arabidopsis seeds. Proc. Natl. Acad. Sci. 97: 10637.

Makeyev E.V. and Bamford D.H. 2002. Cellular RNA-dependent RNA polymerase involved in posttranscriptional gene silencing has two distinct activity modes. Mol. Cell 10: 1417.

Martienssen R. 1998a. Chromosomal imprinting in plants. Curr. Opin. Genet. Dev. 8: 240.

1998b. Transposons, DNA methylation and gene control. Trends Genet. 14: 263.

2003. Maintenance of heterochromatin by RNA interference of tandem repeats. Nat. Genet. 35: 213.

Martienssen R.A. and Colot V. 2001. DNA methylation and epigenetic inheritance in plants and filamentous fungi. Science 293: 1070.

Matzke M., Aufsatz W., Kanno T., Daxinger L., Papp I., Mette M.F., and Matzke A.J. 2004. Genetic analysis of RNA-mediated transcriptional gene silencing. Biochim. Biophys. Acta 1677: 129.

McClintock B. 1957. Controlling elements and the gene. Cold Spring Harbor Symp. Quant. Biol. 21: 197.

Miura A., Yonebayashi S., Watanabe K., Toyama T., Shimada H., and Kakutani T. 2001. Mobilization of transposons by a mutation abolishing full DNA methylation in Arabidopsis. Nature 411: 212.

Mohan K.N., Ray P., and Chandra H.S. 2002. Characterization of the genome of the mealybug Planococcus lilacinus, a model organism for studying whole-chromosome imprinting and inactivation. Genet. Res. 79: 111.

Morgan H.D., Sutherland H.G., Martin D.I., and Whitelaw E. 1999. Epigenetic inheritance at the agouti locus in the mouse. Nat. Genet. 23: 314.

Morris K.V., Chan S.W., Jacobsen S.E., and Looney D.J. 2004. Small interfering RNA-induced transcriptional gene silencing in human cells. Science 305: 1289.

Peaston A.E., Evsikov A.V., Graber J.H., de Vries W.N., Holbrook A.E., Solter D., and Knowles B.B. 2004. Retrotransposons regulate host genes in mouse oocytes and preimplantation embryos. Dev. Cell 7: 597.

Rabinowicz P.D., Palmer L.E., May B.P., Hemann M.T., Lowe S.W., McCombie W.R., and Martienssen R.A. 2003. Genes and transposons are differentially methylated in plants, but not in mammals. Genome Res. 13: 2658.

Ruan K. and Emmons S.W. 1984. Extrachromosomal copies of transposon Tc1 in the nematode Caenorhabditis elegans. Proc. Natl. Acad. Sci. 81: 4018.

Rudenko G.N., Ono A., and Walbot V. 2003. Initiation of silencing of maize $\mathrm{MuDR} / \mathrm{Mu}$ transposable elements. Plant $J$. 33: 1013 .

Saze H., Scheid O.M., and Paszkowski J. 2003. Maintenance of $\mathrm{CpG}$ methylation is essential for epigenetic inheritance during plant gametogenesis. Nat. Genet. 34: 65 .

Schramke V. and Allshire R. 2003. Hairpin RNAs and retrotransposon LTRs effect RNAi and chromatin-based gene silencing. Science 301: 1069.

Sigova A., Rhind N., and Zamore P.D. 2004. A single Argonaute protein mediates both transcriptional and posttranscriptional silencing in Schizosaccharomyces pombe. Genes Dev. 18: 2359.

Sijen T. and Plasterk R.H. 2003. Transposon silencing in the Caenorhabditis elegans germ line by natural RNAi. Nature 426: 310 .

Singer T., Yordan C., and Martienssen R.A. 2001. Robertson's Mutator transposons in A. thaliana are regulated by the chromatin-remodeling gene Decrease in DNA Methylation (DDM1). Genes Dev. 15: 591.

Sleutels F. and Barlow D.P. 2002. The origins of genomic imprinting in mammals. Adv Genet. 46: 119.

Sleutels F., Zwart R., and Barlow D.P. 2002. The non-coding Air RNA is required for silencing autosomal imprinted genes. $\mathrm{Na}$ ture 415: 810. 
Song J.J., Smith S.K., Hannon G.J., and Joshua-Tor L. 2004. Crystal structure of Argonaute and its implications for RISC slicer activity. Science 305: 1434.

Song J.J., Liu J., Tolia N.H., Schneiderman J., Smith S.K., Martienssen R.A., Hannon G.J., and Joshua-Tor L. 2003. The crystal structure of the Argonaute2 PAZ domain reveals an RNA binding motif in RNAi effector complexes. Nat. Struct. Biol. 10: 1026.

Soppe W.J., Jacobsen S.E., Alonso-Blanco C., Jackson J.P., Kakutani T., Koornneef M., and Peeters A.J. 2000. The late flowering phenotype of fwa mutants is caused by gain-of-function epigenetic alleles of a homeodomain gene. Mol. Cell 6: 791.

Soppe W.J., Jasencakova Z., Houben A., Kakutani T., Meister A., Huang M.S., Jacobsen S.E., Schubert I., and Fransz P.F. 2002. DNA methylation controls histone H3 lysine 9 methylation and heterochromatin assembly in Arabidopsis. EMBOJ. 21: 6549 .

Stam M., Belele C., Dorweiler J.E., and Chandler V.L. 2002. Differential chromatin structure within a tandem array $100 \mathrm{~kb}$ upstream of the maize b1 locus is associated with paramutation. Genes Dev. 16: 1906.

Sundaresan V. and Freeling M. 1987. An extrachromosomal form of the Mu transposons of maize. Proc. Natl. Acad. Sci. 84: 4924.

Tabata S., Kaneko T., Nakamura Y., Kotani H., Kato T., Asamizu E., Miyajima N., Sasamoto S., Kimura T., Hosouchi T., Kawashima K., Kohara M., Matsumoto M., Matsuno A., Muraki A., Nakayama S., Nakazaki N., Naruo K., Okumura S., Shinpo S., Takeuchi C., Wada T., Watanabe A., Yamada M., Yasuda M., et al. 2000. Sequence and analysis of chromosome 5 of the plant Arabidopsis thaliana. $\mathrm{Na}$ ture 408: 823 .
Tariq M. and Paszkowski J. 2004. DNA and histone methylation in plants. Trends Genet. 20: 244.

Tariq M., Saze H., Probst A.V., Lichota J., Habu Y., and Paszkowski J. 2003. Erasure of $\mathrm{CpG}$ methylation in Arabidopsis alters patterns of histone $\mathrm{H} 3$ methylation in heterochromatin. Proc. Natl. Acad. Sci. 100: 8823.

Verdel A., Jia S., Gerber S., Sugiyama T., Gygi S., Grewal S.I., and Moazed D. 2004. RNAi-mediated targeting of heterochromatin by the RITS complex. Science 303: 672.

Vielle-Calzada J.P., Thomas J., Spillane C., Coluccio A., Hoeppner M.A., and Grossniklaus U. 1999. Maintenance of genomic imprinting at the Arabidopsis medea locus requires zygotic DDM1 activity. Genes Dev. 13: 2971.

Volpe T.A., Kidner C., Hall I.M., Teng G., Grewal S.I., and Martienssen R.A. 2002. Regulation of heterochromatic silencing and histone H3 lysine-9 methylation by RNAi. Science 297: 1833.

Vongs A., Kakutani T., Martienssen R.A., and Richards E.J. 1993. Arabidopsis thaliana DNA methylation mutants. Science 260: 1926.

Xiao W., Gehring M., Choi Y., Margossian L., Pu H., Harada J.J., Goldberg R.B., Pennell R.I., and Fischer R.L. 2003. Imprinting of the MEA Polycomb gene is controlled by antagonism between MET1 methyltransferase and DME glycosylase. Dev. Cell 5: 891.

Xie Z., Johansen L.K., Gustafson A.M., Kasschau K.D., Lellis A.D., Zilberman D., Jacobsen S.E., and Carrington J.C. 2004. Genetic and functional diversification of small RNA pathways in plants. PLoS Biol. 2: E104.

Zilberman D., Cao X., and Jacobsen S.E. 2003. ARGONAUTE4 control of locus-specific siRNA accumulation and DNA and histone methylation. Science 299: 716. 


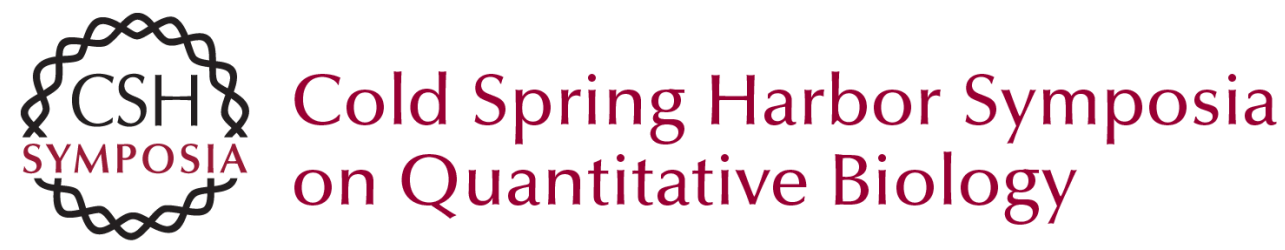

\section{Transposons, Tandem Repeats, and the Silencing of Imprinted Genes}

R. MARTIENSSEN, Z. LIPPMAN, B. MAY, et al.

Cold Spring Harb Symp Quant Biol 2004 69: 371-380

Access the most recent version at doi:10.1101/sqb.2004.69.371

References This article cites 75 articles, 32 of which can be accessed free at: http://symposium.cshlp.org/content/69/371.full.html\#ref-list-1

License

Email Alerting Receive free email alerts when new articles cite this article - sign up in Service the box at the top right corner of the article or click here. 